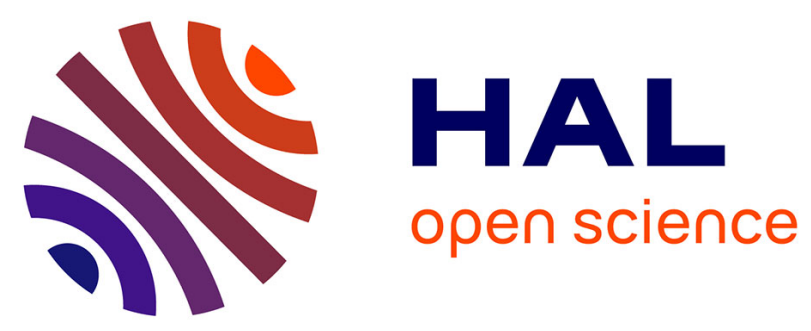

\title{
Sélection pour la résistance à la deltaméthrine d'une souche de Phytoseiulus persimilis Athias- Henriot
}

\author{
Martine Avella, Didier Fournier, Madeleine Pralavorio, Jean-Baptiste Berge
}

\section{To cite this version:}

Martine Avella, Didier Fournier, Madeleine Pralavorio, Jean-Baptiste Berge. Sélection pour la résistance à la deltaméthrine d'une souche de Phytoseiulus persimilis Athias- Henriot. Agronomie, 1985, 5 (2), pp.177-180. hal-00884747

\section{HAL Id: hal-00884747 https://hal.science/hal-00884747}

Submitted on 1 Jan 1985

HAL is a multi-disciplinary open access archive for the deposit and dissemination of scientific research documents, whether they are published or not. The documents may come from teaching and research institutions in France or abroad, or from public or private research centers.
L'archive ouverte pluridisciplinaire HAL, est destinée au dépôt et à la diffusion de documents scientifiques de niveau recherche, publiés ou non, émanant des établissements d'enseignement et de recherche français ou étrangers, des laboratoires publics ou privés. 


\section{Sélection pour la résistance à la deltaméthrine d'une souche de Phytoseiulus persimilis Athias- Henriot}

Martine AVELLA, Didier FOURNIER, Madeleine PRALAVORIO \& Jean-Baptiste BERGE (*)

I.N.R.A., Station de Zoologie et de Lutte biologique

(*) Station de Nématologie, F 06602 Antibes

Dans le cadre d'un programme visant à rendre une souche de Phytoseiulus persimilis Athias-Henriot résistant à l'ensemble des produits phytosanitaires, nous avons sélectionné une souche déjà résistante au méthidathion, pour la résistance à la deltaméthrine. Pour cela, nous avons, à intervalles réguliers, traité la population à une dose mortelle pour $50 \mathrm{p} .100$ des individus. L'acquisition de cette résistance, bien qu'insuffisante pour que les adultes survivent à une application de deltaméthrine sur le terrain, devrait permettre à cette souche d'être moins affectée par des résidus provenant de traitements antérieurs au lâcher.

Mots clés additionnels : Phytoseiidae, Acarina, sélection, pyréthrinoìdes, insecticide, résistance.

In order to make a strain of Phytoseiulus persimilis Athias-Henriot resistant to several pesticides, a strain already resistant to methidathion was selected for deltamethrin resistance. Seven rounds of selection resulted in about a 10-fold increase in LC 50. The level of deltamethrin resistance was too weak for direct use in the field, but it may facilitate establishment of the strain in glasshouses after a deltamethrin application.

Additional key words : Phytoseiidae, acarina, selection, pyrethroid, insecticide, resistance.

\section{INTRODUCTION}

Dans les serres de cultures maraîchères et florales, l'acarien prédateur Phytoseiulus persimilis AthiasHenriot peut être utilisé pour lutter contre l'acarien Tetranychus urticae Koch. Ce ravageur est toutefois rarement le seul sur la culture, aussi l'agriculteur se trouve-t-il souvent obligé d'utiliser des insecticides conjointement à la lutte biologique. Parmi ces insecticides, les pyréthrinoïdes qui sont de plus en plus utilisés sont toxiques pour le prédateur.

Divers essais entrepris pour obtenir des insectes utiles résistants aux pesticides (HOY, 1976), en particulier des phytoseiides, ont été couronnés de certains succès (BEGLYAROV et al., 1978; SCHULTEN \& VAN DE KLASHORST, 1974 ; HOY \& KNOP, 1981 ; ROUSH \& HOY, 1981 ; STRICKLER \& CROFT, 1982). Comme à ce jour on ne dispose pas de souche de $P$. persimilis résistante aux pyréthrinoïdes, notre but était d'en sélectionner une résistant à la deltaméthrine, insecticide couramment employé en France.

L'action de ce pesticide se manifeste d'abord par un effet de choc, « Knock down » ou KD, qui se traduit par une paralysie chez l'insecte ou l'acarien. Dans une certaine échelle de concentration, cet effet est réversible, mais à des concentrations plus élevées, l'effet KD est suivi de mort. Nous avons essayé de préciser les mécanismes de la résistance en étudiant l'influence de cette résistance sur les effets KD et la mortalité. Par la suite, une étude sur la stabilité de la résistance et sur l'effet du produit sur le terrain nous a permis d'apprécier la portée pratique de cette sélection.

\section{MATÉRIEL ET MÉTHODE}

La sélection a porté sur une souche composée d'un mélange de 7 souches originaires de différents laboratoires européens ou récoltées sur le terrain dans le sud est de la France. Ces souches ont été sélectionnées séparément pour la résistance au méthiciathion. Elles ont ensuite été mélangées et la souche en résultant a été sélectionnée pour la résistance à ce même insecticide pendant six mois. La souche que nous avons utilisée est donc résistante au méthidathion et par résistance croisée à d'autres organophosphorés. A la suite 
de cette sélection, cette souche a été lâchée sur le terrain chez divers agriculteurs penclant un été. Elle a alors subi divers traitements insecticides, organophosphorés ou pyréthrinoïdes.

A la fin de la saison, cette souche, récupérée sur le terrain en plusieurs endroits, a été soumise à la sélection pour la résistance à la deltaméthrine. A titre de témoin de comparaison, nous avons aussi utilisé une souche sensible, gardée depuis 13 ans au laboratoire à l'abri de tout traitement. Cette souche était une des 7 souches composant la souche sélectionnée.

L'action létale de la deltaméthrine est déterminée chez des femelles adultes par contact tarsal : des tubes à hémolyse sont traités à l'aide de solutions acétoniques de deltaméthrine de concentrations croissantes. Après séchage, leur face interne est recouverte d'une quantité de produit proportionnelle à la dose utilisée, 5 individus sont alors introduits dans chacun d'entre eux. $P$. persimilis étant très sensible au jeûne, le contrôle doit être fait relativement rapidement, dans les heures qui suivent le traitement. Toutefois la discrimination des individus morts, KD ou vivants, est alors trop difficile. Pour tourner cette difficulté, $4 \mathrm{~h}$ après le début du traitement, le tube est ouvert et joint à une boîte comportant de la nourriture (fig. 1). Les femelles non affectées par le pesticide sont attirées par les tétranyques (SABELIS \& VAN DE BAAN, 1983) et sortent du tube traité, alors que les autres y restent et meurent : $16 \mathrm{~h}$ après, la boîte pourvue de tétranyques contient les femelles vivantes et le tube traité contient uniquement des morts. Cette méthode nous permet d'enregistrer un effet dose/mortalité et par la suite de calculer une dose létale 50 (DL 50), (FISCHER \& YATES, 1963). La sélection est effectuée à cette DL 50, une centaine de femelles étant traitées à cette dose. Les survivants donnent naissance à une population qui, après une vingtaine de jours, soit 2 générations, est suffisamment abondante pour subir une nouvelle sélection. La sélection est poursuivie aussi longtemps que l'on observe une augmentation de la DL 50.

Pour étudier l'effet de la résistance sur le KD, nous avons au temps initial déposé 10 femelles de $P$. persimilis dans un tube à hémolyse préalablement traité comme précédemment. Ce tube est renversé sur une surface encollée. Lorsque 5 des 10 femelles sont tombées, $\mathrm{KD}$, le temps est noté. L'opération est répétée une dizaine de fois par dose et par souche, ce qui permet de calculer un tKD 50 moyen (temps pour lequel 50 p. 100 des femelles sont KD).

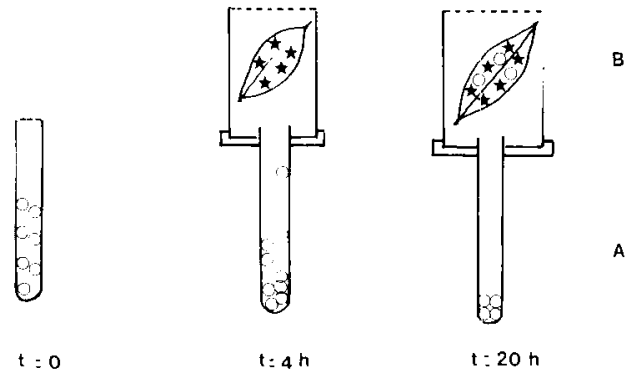

Figure 1

Méthode de test $\mathrm{O}: \mathrm{P}$. persimilis - * : T. urticae ( ) - A : tube traité - $B$ : compartiment non traité.

Test method O : P. persimilis - * : T. urticae ( ) - $A$ : treated tube $B$ : non treated tube.

\section{RÉSULTATS ET DISCUSSION}

Les tests effectués sur la souche témoin et sur la souche de terrain montrent qu'elles présentent des résistances différentes à la deltaméthrine, la $2^{\mathrm{e}}$ étant la plus résistante (fig. 2, droites a et b). Il est vraisemblable que la résistance observée ici a été acquise par les souches de terrain lors des traitements insecticides qu'elles ont subies soit avant, soit après la sélection au méthidathion. En effet, les tests effectués lors de cette sélection ont montré qu'il n'y avait pas de résistance croisée entre cet organophosphoré et la deltaméthrine. La pente moins élevée de la droite de régression concentration/mortalité pour la souche de terrain traduit probablement un polymorphisme génétique plus élevé de cette dernière, une plus grande fréquence des gènes de résistance, ce qui tend à indiquer qu'une sélection est possible.

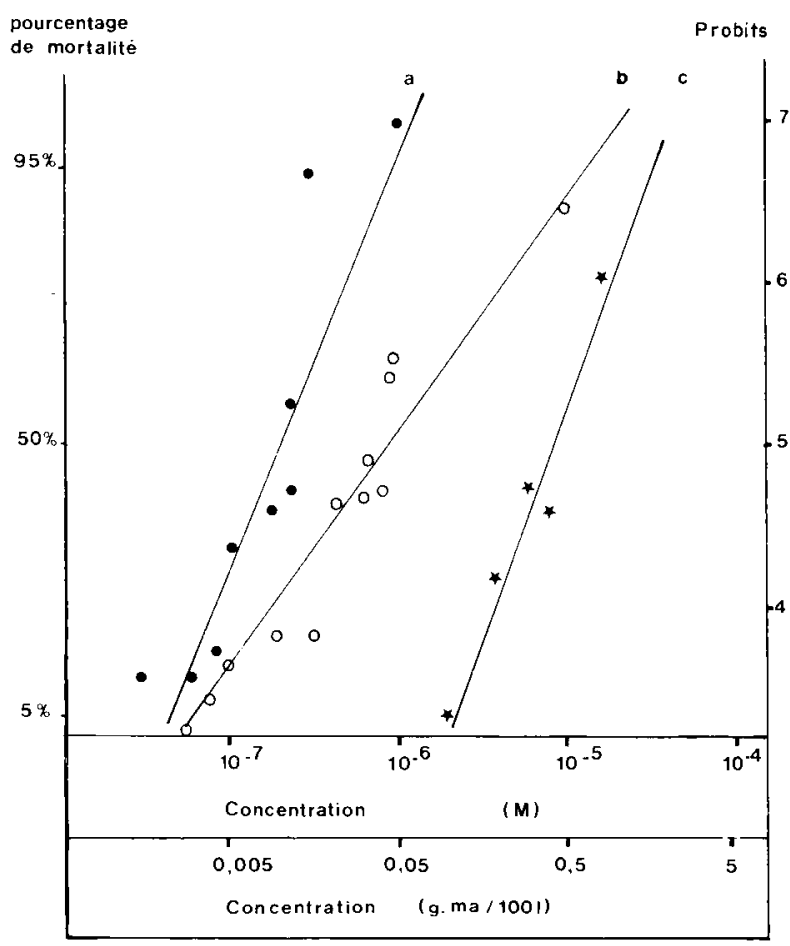

Figure 2

Courbe dose-mortalité pour la deltaméthrine chez les 3 souches de $\mathrm{P}$. persimilis ( $a$ : souche témoin - $b$ : souche de terrain avant sélection à la deltaméthrine $-c$ : souche de terrain après sélection à la deltaméthrine).

Dose-mortality curve for deltamethrin and 3 strains of $\mathrm{P}$. persimilis ( $a$ : reference strain - $b:$ field strain before selection $-c:$ field strain after selection).

La courbe d'acquisition de la résistance pour la souche de terrain suit le modèle habituel : l'évolution est tout d'abord lente puis s'accélère jusqu'à un plateau (fig. 3 ). Ce dernier est atteint après 6 sélections successives correspondant à 12 générations, ce qui est relativement rapide. Il présente toutefois un rapport de résistance faible, de l'ordre de 10 (fig. 2, droites b et c). L'étude comparée des tKD 50 moyens des souches, avant et après sélection à la deltaméthrine (fig. 4), montre que l'on retrouve une différence de sensibilité de 1 à 10 pour les faibles concentrations allant de $10^{-7} \mathrm{M}$ à $10^{-6} \mathrm{M}$, concentrations qui entraînent une mortalité partielle (fig. 2, droites b et c). Par contre, aux doses plus élevées $\left(10^{-5} \mathrm{M}\right.$ à $\left.10^{-3} \mathrm{M}\right)$, les 


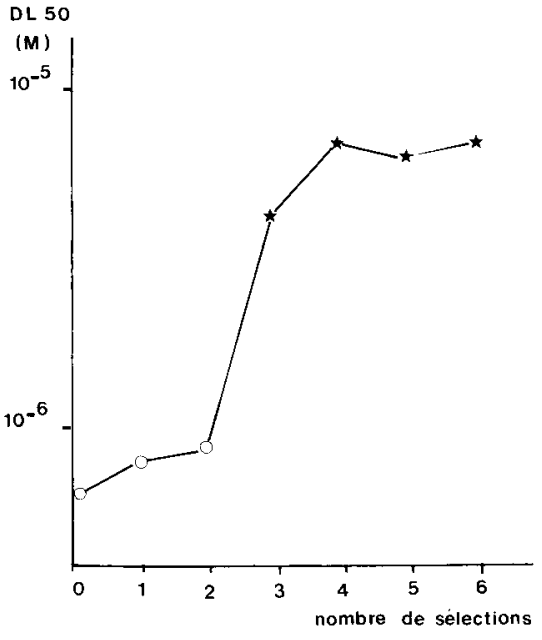

Figure 3

Changement de la DL 50 du cours de la sélection de $\mathrm{P}$. persimilis vis-à-vis de la deltaméthrine.

Change in LC 50 during the selection of $\mathrm{P}$. persimilis for deltamethrin resistance.

tKD 50 sont identiques pour les 2 souches. Ce résultat semble autoriser à expliquer la résistance par un phénomène de métabolisation : les individus résistants dégraderaient la deltaméthrine plus vite que les sensibles. Cette différence de dégradation, négligeable aux fortes concentrations, pourrait jouer un rôle important aux faibles concentrations. Nous avons vérifié la résistance de la souche sélectionnée après 6 mois de suspension de la sélection. La DL 50 obtenue $\left(7,9 \cdot 10^{-6} \mathrm{M}\right)$ n'est pas significativement différente de celle obtenue en fin de sélection : la résistance demeure donc stable durant cette période. Par contre, le traitement en laboratoire bien que partiel, limité aux tarses pendant un temps déterminé, nous montre que la dose habituelle d'emploi $(1 \mathrm{~g} \mathrm{~m}$.a. $/ 100$ litres : $2.10^{-5} \mathrm{M}$ ) entraîne une mortalité totale. Ce résultat s'est retrouvé sur le terrain lorsque, après avoir lâché une population issue de la souche sélectionnée en serre, elle a été complètement éliminée de la culture par des applications de deltaméthrine à cette même dose d'emploi.

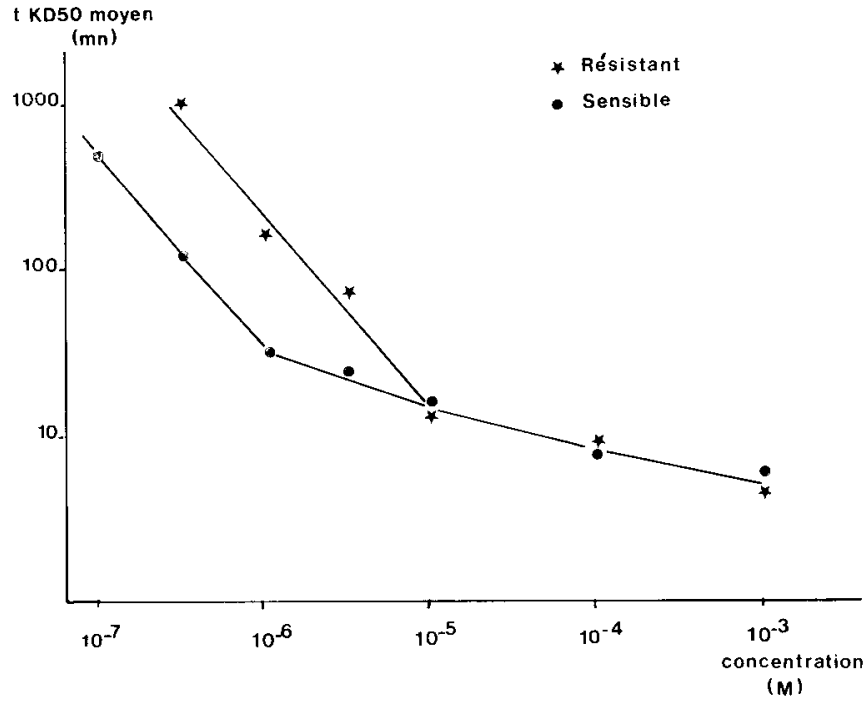

Figure 4

Effet de la sélection sur la variation du $t K D 50$ moyen en fonction de la dose $1^{*}$ : souche sélectionnée à la deltaméthrine - $\bullet$ : souche de terrain avant la sélection à la deltaméthrine).

Effect of selection on time for $50 \%$ knock-down in relation to dose $1^{*}:$ deltamethrin selected strain - $\bullet$ field strain before selection)

\section{CONCLUSION}

Le taux de résistance acquis est relativement faible, 7 fois en ce qui concerne la simple sélection au pesticide (fig. 2 , droites b et c) et $\mathbf{3 6}$ fois lorsqu'on fait intervenir un ensemble de procédés tendant à l'amélioration génétique (mélange, lâcher et sélection, fig. 2, droites a et c). L’origine métabolique de la résistance, postulée à la suite de la comparaison des tKD 50 (fig. 3) peut expliquer cette faible réponse à la sélection. En effet, pour les pyréthrinoïdes une différence de métabolisation n'entraîne souvent qu'une résistance faible comparée à celle qui résulte d'une insensibilité se situant au niveau de l'action sur la membrane nerveuse. Les essais de sélection du même type, effectués sur des phytoséiides ont donné des résultats variables : les facteurs de résistance atteints varient de 0 à 363 fois selon l'espèce, le pesticide et, vraisemblablement, la méthode de test employée (tabl. 1). En géné-

TABLEAU 1

Sélection de Phytoseiidae pour la résistance aux pesticides. Selection of Phytoseiidae for pesticide resistance.

\begin{tabular}{|c|c|c|c|c|c|}
\hline Espèces & Produits & $\begin{array}{l}\text { Facteurs } \\
\text { de } \\
\text { résistance }\end{array}$ & $\begin{array}{l}\text { Nombre } \\
\text { de } \\
\text { sélections }\end{array}$ & $\begin{array}{l}\text { Tolérance } \\
\text { sur le } \\
\text { terrain } \\
(1)\end{array}$ & Références \\
\hline Amblyseius fallacis Garman & carbaryl & 25 à 77 & - & + & Croft \& MEyer (1973) \\
\hline Amblyseius fallacis Garman & azinphosméthyl & 337 à 363 & - & + & Croft \& Meyer (1973) \\
\hline Amblyseius fallacis Garman & perméthrine & 64 & 12 & + & STRICKLER \& CROFT (1982) \\
\hline Metaseiulus occidentalis Nesbitt. & méthomyl & 12 & 12 & - & ROUSH \& HoY (1981), HoY (1981) \\
\hline Metaseiulus occidentalis Nesbitt. & carbaryl & - & $15-19$ & + & RousH \& HOY (1981), HoY (1981) \\
\hline Metaseiulus occidentalis Nesbitt. & perméthrine & 10 & 18 & + & HOY \& KNOP (1981), HOY (1981) \\
\hline Phytoseiulus persimilis Athias-Henriot & ethion & 4 & 9 & + & BEGLYAROV et al. (1978) \\
\hline Phytoseiulus persimilis Athias-Henriot & parathion & 100 & 8 & + & $\begin{array}{l}\text { SCHULTEN \& VAN DE KLASHORST (1974), } \\
\text { SCHULTEN et al. (1976) }\end{array}$ \\
\hline Phytoseiulus persimilis Athias-Henriot & methidathion & 120 & 50 & + & FOURNIER (1981) \\
\hline Phytoseiulus persimilis Athias-Henriot & D.D.V.P. & 4 & 5 & & FOURNIER et al. (non publié) \\
\hline Phytoseiulus persimilis Athias-Henriot & roténone & 0 & 5 & - & FOURNIER et al. (non publié) \\
\hline
\end{tabular}

(1) Populations survivant $(+)$ ou non $(-)$ à la dose habituelle d'emploi sur le terrain.

(1) Population surviving a treatment $(+)$ or not $(-)$ in the field at the usual dose. 
ral, les niveaux de résistance atteints sont assez élevés pour permettre aux populations de survivre à des applications de pesticides aux doses habituelles d'emploi. Si nous n'avons pas obtenu un tel succès, il semble que ce soit la conséquence de la forte toxicité de la deltaméthrine vis-à-vis de $P$. persimilis. Malgré ce résultat médiocre, il est vraisemblable que la souche sélectionnée est moins sensible aux résidus de deltaméthrine provenant d'un traitement antérieur au lâcher, puisqu'elle se trouve alors en face de très faibles concentrations du pesticide. Il est par ailleurs vraisemblable que la sélection à la deltaméthrine occasionne des résistances croisées à d'autres pyréthrinoïdes, mais ceci demande à être vérifié. Quoi qu'il en soit, le meilleur moyen envisagé à court terme pour augmenter la résistance à la deltaméthrine semble être d'alterner des périodes de sélection avec des périodes de lâcher dans des zones traitées avec différents pro- duits phytosanitaires, zones choisies de telle sorte que $P$. persimilis y soit indigène. Cette technique devrait permettre d'augmenter le polymorphisme de la souche sélectionnée par croisement avec des souches sauvages élargissant ainsi les possibilités de réponse à la sélection. A plus long terme, il est possible d'envisager un transfert de gènes à partir d'une autre espèce très résistante. Une telle technique permettrait de se libérer du manque de variabilité naturelle en ce qui concerne les gènes impliqués dans la résistance.

Reçu le 5 mars 1984 Accepté le 10 octobre 1984

\section{REMERCIEMENTS}

Nous remercions Monsieur CARLEs et la firme PROCIDA pour leur aide dans la réalisation de ce travail.

\section{RÉFÉRENCES BIBLIOGRAPHIQUES}

Beglyarov G. A., Zil'bermints I. V., Petrushkov A. Z., 1978. « Une race de Phytoseiulus résistante aux insecto-acaricides. Moyens de l'utiliser dans un système de lutte intégrée contre les ravageurs sous serre ». In Biologicheskij metod bor' by s wreditelyami i Boleznyami rastenij v zakrytom grunte, Moskva, kolos, 51-63 (en russe).

Croft B. A., Meyer R. H., 1973. Carbamate and organophosphorus resistance patterns in populations of Amblyseius fallacis. Environ. Entomol. 2, (4), 691-694.

Fischer R. A., Yates Y., 1963. Statistical tables for biological, agricultural and medical research. 6th ed. revised and enlarged. Olivier and Boyd ed., Edimbourg, 146 p.

Fournier D., 1981. Acquisition de la résistance au methidathion chez Phytoseiulus persimilis, A. H., Evolution, mécanismes et répercussions. Thèse de $3^{\mathrm{e}}$ cycle, Univ. Paris VI, $44 \mathrm{p}$.

Hoy M. A., 1976. Genetic improvement of insects : fact or fantasy. Environ. Entomol., 5, 831-833.

Hoy M. A., 1981. Recent advances in knowledge of Phytoseiidae. Proc. of a formal conference of the acarology society of America, San Diego, déc. 1981, 72-89.
Hoy M. A., Knop N. F., 1981. Selection for and genetic analysis of permethrin resistance in Metaseiulus occidentalis : genetic improvement of a biological control agent. Entomol. Exp. Appl., 30, 10-18.

Roush R. T., Hoy M. A., 1981. Genetic improvement of Metaseiulus occidentalis, selection with methomyl, dimethoate and carbaryl and genetic analysis of carbaryl resistance. $J$. econ. Entomol., 74 (2), 138-141.

Sabelis M. W., Van de Baan H. E., 1983. Location of distant spider mite colonies by phytoseiid predators : demonstration of specific kairomones emitted by Tetranychus urticae and Panonychus ulmi. Entomol. Exp. Appl., 33, 303-314.

Schulten G. G. M., Van de Klashorst G., 1974. Genetics of resistance to parathion and demeton-S-methyl in Phytoseiulus persimilis A. H., Proc. Int. Congr. Acarology, 519-524.

Schulten G. G. M., Van de Klashorst G., Russel V. M., 1976. Resistance of Phytoseiulus persimilis A. H. (Acari : Phytoseiidae) to some insecticides. Z. angew. Entomol., 80, 337-341.

Strickler K., Croft B. A., 1982. Selection for permethrin resistance in the predatory mite Amblyseius fallacis. Entomol. Exp. Appl., 31, 339-345. 